\title{
A New Algorithm for Joint Range-DOA-Frequency Estimation of Near-Field Sources
}

\author{
Jian-Feng Chen \\ Key Lab for Radar Signal Processing, Xidian University, Xi'an 710071, China \\ Email:strake2003@yahoo.com.cn \\ Xiao-Long Zhu \\ Key Lab for Radar Signal Processing, Xidian University, Xi'an 710071, China \\ Department of Automation, Tsinghua University, Beijing 100084, China \\ Email:xlzhu_dau@mail.tsinghua.edu.cn

\section{Xian-Da Zhang} \\ Department of Automation, Tsinghua University, Beijing 100084, China \\ Email: zxd-dau@mail.tsinghua.edu.cn
}

Received 20 December 2002; Revised 29 August 2003; Recommended for Publication by Zhi Ding

This paper studies the joint estimation problem of ranges, DOAs, and frequencies of near-field narrowband sources and proposes a new computationally efficient algorithm, which employs a symmetric uniform linear array, uses eigenvalues together with the corresponding eigenvectors of two properly designed matrices to estimate signal parameters, and does not require searching for spectral peak or pairing among parameters. In addition, the proposed algorithm can be applied in arbitrary Gaussian noise environment since it is based on the fourth-order cumulants, which is verified by extensive computer simulations.

Keywords and phrases: array signal processing, DOA estimate, range estimate, frequency estimate, fourth-order cumulant.

\section{INTRODUCTION}

In array signal processing, there exist many methods to estimate the directions of arrival (DOAs) of far-field sources impinging on an array of sensors [1], such as MUSIC, ESPRIT, and so forth. Most of these methods make an assumption that sources locate relatively far from the array, and thus the wavefronts from the sources can be regarded as plane waves. Based on this assumption, each source location can be characterized by a single DOA [1]. When the source is close to the array, namely, in the near-field case, however, this assumption is no longer valid. The near-field sources must be characterized by spherical wavefronts at the array aperture and need to be localized both in range and in DOA $[2,3,4]$. The near-field situation can occur, for example, in sonar, electronic surveillance, and seismic exploration.

To deal with the joint range-DOA estimation problem of near-field sources, many approaches have been presented $[2,3,4,5,6,7,8,9]$. The maximum likelihood estimator proposed in [2] has optimal statistical properties, but it needs multidimensional search and is highly nonlinear. Huang and Barkat [3] and Jeffers et al. [4] extended the conventional one-dimensional (1D) MUSIC method to the two-dimensional (2D) ones for range and DOA estimates. Since 2D MUSIC requires an exhaustive 2D search, their approaches are computationally inefficient. To avoid multidimensional search, Challa and Shamsunder [7] developed a total least squares ESPRIT-like algorithm which applies the fourth-order cumulants to estimate the DOAs and ranges of near-field sources. Nevertheless, it still requires heavy computations to construct a higher-dimensional cumulant matrix in order to obtain the so-called signal subspace, and the computational load becomes even intolerable when the number of sensors is very large. More recently, a weighted linear prediction method for near-field source localization was presented in [9], but it needs additional computation to solve the pairing problem among parameters in the case of multiple sources.

All the methods above assume that the carrier frequencies are available. If the carrier frequencies are unknown, the location problem of near-field sources is actually a threedimensional (3D) one because three parameters of the DOA, range, and the associated frequency of each source should be estimated and paired correctly. This paper proposes a 


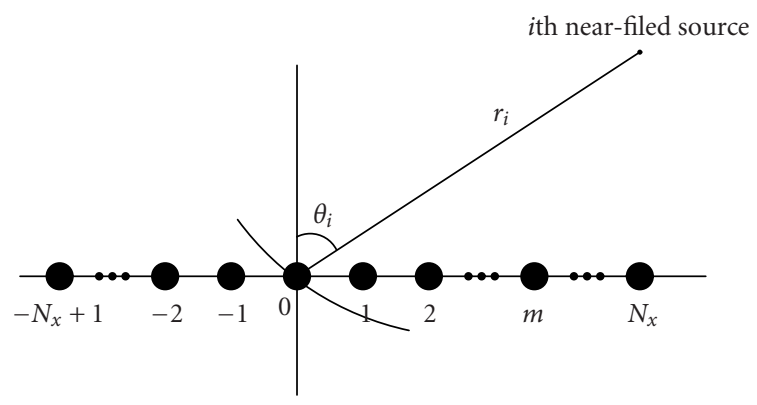

FIgURE 1: Sensor configuration of near-field sources.

computationally efficient algorithm for joint estimation of the DOA, range, and frequency of each near-field source. Without constructing the higher-dimensional cumulant matrix, the proposed algorithm applies a symmetric uniform linear array and uses eigenvalues together with the corresponding eigenvectors of two properly designed matrices to jointly estimate signal parameters, and it does not require any spectral peak searching since the parameters are automatically paired.

This paper is organized as follows. Section 2 introduces the signal model and Section 3 develops a new algorithm. In Section 4 , a series of computer simulations are presented to demonstrate the effectiveness of the proposed algorithm, and finally, conclusions are made in Section 5.

\section{PROBLEM FORMULATION}

Consider the narrowband model for array processing of near-field sources, as shown in Figure 1. Suppose that there are $K$ sources of interest with complex baseband representations $s_{i}(t), i=1, \ldots, K$. Let the band of interest have a center frequency $f_{c}$ and the $i$ th source has a carrier frequency $f_{c}+f_{i}$. After demodulation to intermediate frequency, the signal due to the $i$ th source is $e^{j 2 \pi f_{i} t} s_{i}(t)$ and the signal received at the $m$ th antenna is

$$
x_{m}(t)=\sum_{i=1}^{K} s_{i}(t) e^{j 2 \pi f_{i} t} e^{j \tau_{m i}}+z_{m}(t), \quad-N_{x}+1 \leqslant m \leqslant N_{x},
$$

in which $z_{m}(t)$ is the additive noise, $f_{i}$ is the frequency of the $i$ th source, and $\tau_{m i}$ is the phase difference in radians between the $i$ th source signal arriving at sensor $m$ and that at the reference sensor 0 .

Applying the Fresnel approximation, one has the phase difference $\tau_{m i}$ as follows $[2,3,4,5,6]$ :

$$
\begin{aligned}
\tau_{m i} & =\frac{2 \pi r_{i}}{\lambda_{i}}\left(\sqrt{1+\frac{m^{2} d^{2}}{r_{i}^{2}}-\frac{2 m d \sin \theta_{i}}{r_{i}}}-1\right) \approx \gamma_{i} m+\phi_{i} m^{2}, \\
\gamma_{i} & =-2 \pi \frac{d}{\lambda_{i}} \sin \left(\theta_{i}\right), \\
\phi_{i} & =\pi \frac{d^{2}}{\lambda_{i} r_{i}} \cos ^{2}\left(\theta_{i}\right),
\end{aligned}
$$

where $d$ is the interelement spacing of the uniform linear array, while $\lambda_{i}, r_{i}$, and $\theta_{i}$ are the wavelength, range, and bearing of the $i$ th source, respectively. denote

Sample the received signals at proper rate $f=1 / T_{s}$ and

$$
\begin{aligned}
& \mathbf{x}(k)=\left[x_{-N_{x}+1}\left(k T_{s}\right), \ldots, x_{0}\left(k T_{s}\right), \ldots, x_{N_{x}}\left(k T_{s}\right)\right]^{T}, \\
& \mathbf{z}(k)=\left[z_{-N_{x}+1}\left(k T_{s}\right), \ldots, z_{0}\left(k T_{s}\right), \ldots, z_{N_{x}}\left(k T_{s}\right)\right]^{T}, \\
& \mathbf{s}(k)=\left[s_{1}\left(k T_{s}\right) e^{j \omega_{1} k}, \ldots, s_{K}\left(k T_{s}\right) e^{j \omega_{K} k}\right]^{T}
\end{aligned}
$$

in which the superscript $T$ denotes transpose and $\omega_{i}=$ $2 \pi f_{i} T_{s}$, then (1) can be written, in a matrix form, as

$$
\mathbf{x}(k)=\mathbf{B s}(k)+\mathbf{z}(k),
$$

where $\mathbf{B}$ is a $2 N_{x} \times K$ matrix with the $i$ th column vector given by

$$
\begin{aligned}
\mathbf{b}_{i}\left(\theta_{i}, r_{i}\right)= & {\left[e^{j\left(-N_{x}+1\right) \gamma_{i}+j\left(-N_{x}+1\right)^{2} \phi_{i}}, \ldots,\right.} \\
& \left.e^{j\left(-\gamma_{i}+\phi_{i}\right)}, 1, e^{j\left(\gamma_{i}+\phi_{i}\right)}, \ldots, e^{j N_{x} \gamma_{i}+j N_{x}^{2} \phi_{i}}\right]^{T} .
\end{aligned}
$$

The objective of this paper is to deal with the joint estimation problem of the range $r_{i}$, the bearing $\theta_{i}$, and the frequency $f_{i}$. For this purpose, the following assumptions are made:

(A1) the source signals $s_{1}(t), \ldots, s_{K}(t)$ are statistically mutually independent, non-Gaussian, narrowband stationary processes with nonzero kurtoses;

(A2) the sensor noise $z_{m}(t)$ is zero-mean (white or colored) Gaussian signal and independent of the source signals;

(A3) the range parameters of the sources are different from each other, that is, $\phi_{i} \neq \phi_{j}$ for $i \neq j$;

(A4) the array is a uniform linear array with spacing $d \leq$ $\lambda_{i} / 4, i=1, \ldots, K$

(A5) the array is a symmetric array with $2 N_{x}$ antenna sensors and $N_{x}>K$.

\section{A NEW JOINT ESTIMATION ALGORITHM FOR 3D PARAMETERS}

To develop a new joint estimation algorithm, we begin with the fourth-order cumulant matrix $\mathbf{C}_{1}$, the $(m, n)$ th element of which is defined by

$$
\begin{gathered}
\mathrm{C}_{1}(m, n) \triangleq \operatorname{cum}\left\{x_{m}^{*}(k), x_{m+1}(k), x_{n+1}^{*}(k), x_{n}(k)\right\}, \\
0 \leqslant m, n \leqslant N_{x}-1,
\end{gathered}
$$

where the superscript $*$ denotes complex conjugate. Substituting (1) into (8) and using the multilinearity property of cumulant together with the assumptions (A1) and (A2), straightforward but slightly tedious manipulations yield [8]

$$
\mathbf{C}_{1}(m, n)=\sum_{i=1}^{K} c_{4 s_{i}} e^{j 2(m-n) \phi_{i}}
$$


in which $c_{4 s_{i}}=\operatorname{cum}\left\{\left|s_{i}(k)\right|^{4}\right\}$ denotes the (nonnormalized) kurtosis of $s_{i}(k)$. Let $\mathbf{C}_{4 s}=\operatorname{diag}\left[c_{4 s_{1}}, \ldots, c_{4 s_{k}}\right]$ be a diagonal matrix composed of the source kurtoses; we have

$$
\mathbf{C}_{1}=\mathbf{A C}_{4 s} \mathbf{A}^{H},
$$

where the superscript $H$ denotes Hermitian transpose, $\mathbf{A}=$ $\left[\mathbf{a}_{1}, \ldots, \mathbf{a}_{K}\right]$ is an $N_{x} \times K$ matrix, and

$$
\mathbf{a}_{i}=\left[1, e^{j 2 \phi_{i}}, \ldots, e^{j 2\left(N_{x}-1\right) \phi_{i}}\right]^{T}, \quad i=1, \ldots, K .
$$

Since all the source signals are assumed to have nonzero kurtoses, $\mathbf{C}_{4 s}$ is an invertible diagonal matrix. Additionally, due to (A3), different sources have different range parameters, $\mathbf{A}$ is of full column rank. Hence, $\mathbf{C}_{1}$ is an $N_{x} \times N_{x}$ matrix with rank $K$, and it is not of full rank for the assumption (A5) that $K<N_{x}$

Let $\left\{\rho_{1}, \ldots, \rho_{K}\right\}$ and $\left\{\mathbf{v}_{1}, \ldots, \mathbf{v}_{K}\right\}$ be the nonzero eigenvalues and the corresponding eigenvectors of $\mathbf{C}_{1}$, respectively, that is, $\mathbf{C}_{1}=\sum_{i=1}^{K} \rho_{i} \mathbf{v}_{i} \mathbf{v}_{i}^{H}$; we may obtain the pseudoinverse matrix of $\mathbf{C}_{1}$, denoted as $\mathbf{C}_{1}^{\dagger}$, and

$$
\mathbf{C}_{1}^{\dagger}=\sum_{i=1}^{K} \rho_{i}^{-1} \mathbf{v}_{i} \mathbf{v}_{i}^{H} .
$$

Due to (10), $\mathbf{A}$ has the same column span as $\mathbf{V}=\left[\mathbf{v}_{1}, \ldots, \mathbf{v}_{K}\right]$, and thus the projection of $\mathbf{a}_{i}$ onto $\operatorname{span}\left\{\mathbf{v}_{1}, \ldots, \mathbf{v}_{K}\right\}$ equals $\mathbf{a}_{i}$, that is, $\mathbf{V V}^{H} \mathbf{A}=\mathbf{A}$. Therefore, it holds that

$$
\begin{aligned}
\mathbf{C}_{1} \mathbf{C}_{1}^{\dagger} \mathbf{A} & =\sum_{i=1}^{K} \rho_{i} \mathbf{v}_{i} \mathbf{v}_{i}^{H} \cdot \sum_{p=1}^{K} \rho_{p}^{-1} \mathbf{v}_{p} \mathbf{v}_{p}^{H} \cdot \mathbf{A} \\
& =\mathbf{V} \mathbf{V}^{H} \mathbf{A}=\mathbf{A} .
\end{aligned}
$$

Furthermore, for different sensor lags, we define

$$
\begin{aligned}
& \mathrm{C}_{2}(m, n) \triangleq \operatorname{cum}\left\{x_{m-1}^{*}(k), x_{m}(k), x_{-n}^{*}(k), x_{1-n}(k)\right\}, \\
& \mathrm{C}_{3}(m, n) \triangleq \operatorname{cum}\left\{x_{m}^{*}(k+1), x_{m+1}(k), x_{n+1}^{*}(k), x_{n}(k)\right\},
\end{aligned}
$$

and similar to (10), we can show that

$$
\begin{aligned}
& \mathbf{C}_{2}=\mathbf{A C}_{4 s} \boldsymbol{\Omega}^{H} \mathbf{A}^{H}, \\
& \mathbf{C}_{3}=\mathbf{A C}_{4 s} \boldsymbol{\Lambda}^{H} \mathbf{A}^{H},
\end{aligned}
$$

where the narrowband assumption, that is, $s_{i}(k) \approx s_{i}(k+1)$, is used [10] and

$$
\begin{aligned}
\boldsymbol{\Omega} & =\operatorname{diag}\left[e^{-j 2 \gamma_{1}}, \ldots, e^{-j 2 \gamma_{K}}\right], \\
\boldsymbol{\Lambda} & =\operatorname{diag}\left[e^{j \omega_{1}}, \ldots, e^{j \omega_{K}}\right] .
\end{aligned}
$$

Clearly, both $\boldsymbol{\Omega}$ and $\boldsymbol{\Lambda}$ are of full rank, so $\mathbf{C}_{2}$ and $\mathbf{C}_{3}$ have the same rank $K$ as $\mathbf{C}_{1}$.

In what follows, we apply (10), (15), and (16) to derive a new algorithm for joint estimation of the range, DOA, and frequency parameters. For convenience of statement, we denote

$$
\begin{aligned}
& \mathbf{C}=\mathbf{C}_{2}^{H} \mathbf{C}_{1}^{\dagger}, \\
& \overline{\mathbf{C}}=\mathbf{C}_{3}^{H} \mathbf{C}_{1}^{\dagger} .
\end{aligned}
$$

Postmultiplying both sides of (19) by A, and applying (15), we obtain

$$
\mathbf{C A}=\mathbf{A} \boldsymbol{\Omega} \mathbf{C}_{4 s} \mathbf{A}^{H} \mathbf{C}_{1}^{\dagger} \mathbf{A} .
$$

On the other hand, since $\mathbf{A}$ is of full column rank, from (10) we have

$$
\mathbf{C}_{4 s} \mathbf{A}^{H}=\left(\mathbf{A}^{H} \mathbf{A}\right)^{-1} \mathbf{A}^{H} \mathbf{C}_{1} .
$$

Therefore, substituting (22) and (13) into (21) results in

$$
\mathrm{CA}=\mathrm{A} \Omega .
$$

Similarly, it is not difficult to show that

$$
\overline{\mathbf{C A}}=\mathbf{A} \Lambda .
$$

Since from (23) and (24), it can be inferred that the matrices $\mathbf{C}$ and $\overline{\mathbf{C}}$ have the same ranks $K$ and the same eigenvectors, we have the following eigendecompositions:

$$
\begin{aligned}
& \mathbf{C}=\sum_{i=1}^{K} \varphi_{i} \mathbf{u}_{i} \mathbf{u}_{i}^{H}, \\
& \overline{\mathbf{C}}=\sum_{i^{\prime}=1}^{K} \varrho_{i^{\prime}} \mathbf{u}_{i^{\prime}} \mathbf{u}_{i^{\prime}}^{H} .
\end{aligned}
$$

Based on (18), (24), and (26), we obtain the estimate of the frequency, given by

$$
\widehat{\omega}_{i}=\operatorname{angle}\left(\varrho_{i}\right),
$$

where angle $(\cdot)$ denotes the phase angle operator.

According to the assumption (A4), that is, $d \leqslant \lambda_{i} / 4$, (3) implies $-\pi \leqslant 2 \gamma_{i} \leqslant \pi$. Hence, we have from (17), (23), and (25) that angle $\left(\varphi_{i}\right)=-2 \gamma_{i}$. Substituting this equation into (3), we get the estimated DOA as

$$
\hat{\theta}_{i}=\sin ^{-1}\left(\frac{\lambda_{i}}{4 \pi d} \cdot \operatorname{angle}\left(\varphi_{i}\right)\right) .
$$

Additionally, (23), (24), (25), and (26) indicate that A has the same column span as $\mathbf{U}=\left[\mathbf{u}_{1}, \ldots, \mathbf{u}_{K}\right]$, that is, $\operatorname{span}\left\{\mathbf{a}_{1}, \ldots, \mathbf{a}_{K}\right\}=\operatorname{span}\left\{\mathbf{u}_{1}, \ldots, \mathbf{u}_{K}\right\}$, therefore, $\mathbf{a}_{i}$ can be estimated by the associated eigenvector $\mathbf{u}_{i}$. Mimicking [11], one may obtain $\hat{\phi}_{i}$ by minimizing a least squares cost function $\sum_{m=1}^{N_{x}-1}\left(m \phi_{i}-\operatorname{angle}\left(u_{i}(m+1) / u_{i}(1)\right)\right)^{2}$ given by

$$
\hat{\phi}_{i}=\frac{12}{N_{x}\left(N_{x}-1\right)\left(2 N_{x}-1\right)} \sum_{m=1}^{N_{x}-1} m \text { angle }\left(\frac{u_{i}(m+1)}{u_{i}(1)}\right) .
$$


Once $\hat{\theta}_{i}$ and $\hat{\phi}_{i}$ are available, the range $r_{i}$ can be estimated using (4), yielding

$$
\hat{r}_{i}=\pi \frac{d^{2}}{\lambda_{i} \hat{\phi}_{i}} \cos ^{2}\left(\hat{\theta}_{i}\right)
$$

For the proposed algorithm, we point out that, since the eigenvectors (associated with the $K$ nonzero eigenvalues) of $\mathbf{C}$ are easily matched with those of $\overline{\mathbf{C}}$ by contrasting the two sets of eigenvectors [12], while they can be both considered as estimates of the $K$ column vectors of $\mathbf{A}$, it is easy to determine the correct pairing of the range, DOA, and frequency parameters of each source.

Finally, it is helpful to compare the proposed algorithm to the ESPRIT-like one [7]. Both methods require constructing cumulant matrices, but they estimate the DOA and range parameters in different ways. Besides the eigenvalues, the eigenvectors are also used in this paper. More importantly, the proposed algorithm employs ably the narrowband assumption of the sources to estimate the frequencies, and it need not construct the higher-dimensional cumulant matrix, which takes advantages over the one presented in [7]. Concerning the computational complexity, we ignore the same computational load of the two methods that is comparatively small (e.g., calculations involved in (19) and (20) in this paper and similar operations in the ESPRIT-like one) and consider the major part, namely, multiplications involved in calculating the cumulant matrices and in performing the eigendecompositions, our algorithm requires $27\left(2 N_{x}+1\right)^{2} M+(4 / 3) N_{x}^{3}$, while the ESPRIT-like one requires $36\left(2 N_{x}+1\right)^{2} M+(4 / 3)\left(3 N_{x}\right)^{3}$, where $M$ is the number of snapshots and $N_{x}$ is the number of sensor. Clearly, the proposed algorithm is computationally more efficient, and in general cases, $M \gg N_{x}$, it has the computational load, at most, 75 percent of the ESPRIT-like one [7].

\section{SIMULATION RESULTS}

To verify the effectiveness of the proposed algorithm, we consider a uniform linear array consisting of $N=14$ sensors with element spacing $d=\min \left(\lambda_{i} / 4\right)$. Two equi-power statistically independent sources impinge on the linear array, and the received signals are polluted by zero-mean additive Gaussian noises. We assume that the two sources are narrowband (bandwidth $=25 \mathrm{kHz}$ ) amplitude modulated signals with the center frequency equal to $2 \mathrm{MHz}$ and $4 \mathrm{MHz}$, respectively. The data are sampled at a rate of $20 \mathrm{MHz}$. The performance is measured by the estimated root mean square error (RMSE):

$$
E_{\mathrm{RMSE}}=\sqrt{\frac{1}{N_{e}} \sum_{i=1}^{N_{e}}\left(\hat{\alpha}_{i}-\alpha_{\text {true }}\right)^{2}}
$$

in which $\hat{\alpha}_{i}$ denotes estimate of the true parameter $\alpha_{\text {true }}$ obtained in the $i$ th run, while $N_{e}$ is the total number of MonteCarlo runs.

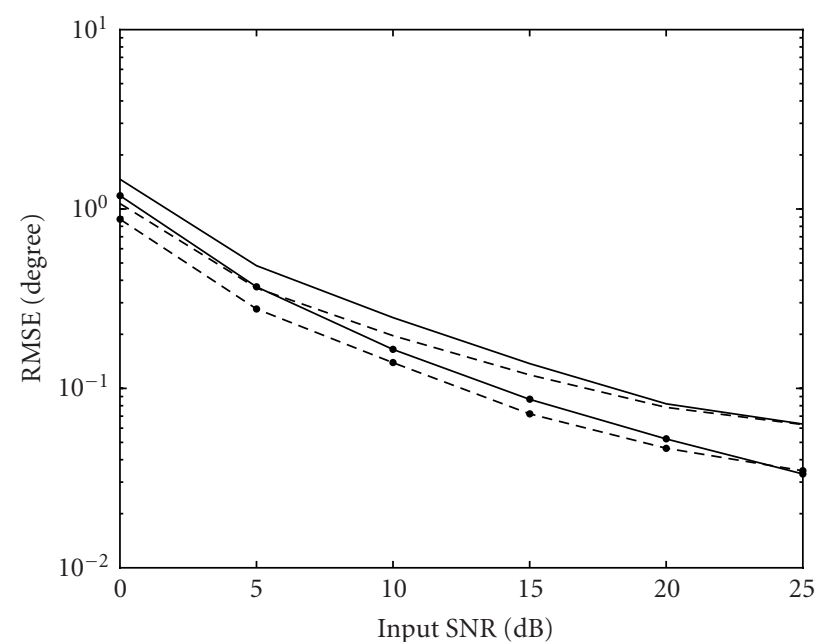

- The proposed method, source 1
- The proposed method, source 2
-- The ESPRIT-like method, source 1
-- The ESPRIT-like method, source 2

Figure 2: The RMSE of the estimated DOA over 500 Monte Carlo runs versus the input SNR; 14 sensors and 1000 snapshots are used and the two equi-power sources approach the array from $38^{\circ}$ and $20^{\circ}$, respectively.

For comparison, we execute the ESPRIT-like algorithm proposed in [7] at the same time and simulate two different cases.

In the first experiment, the first source locates at $\theta_{1}=38^{\circ}$ with range $r_{1}=1.3 \lambda_{1}$ and the other locates at $\theta_{2}=20^{\circ}$ with range $r_{2}=0.65 \lambda_{2}$. The RMSE of range parameter is normalized (divided) by the signal wavelength $\lambda$. The number of snapshots is set to 1000 and the signal-to-noise ratio (SNR) varies from $0 \mathrm{~dB}$ to $25 \mathrm{~dB}$. The additive Gaussian noise may be white or colored. Since the results are alike, we simply consider the colored Gaussian noise as below:

$$
z(k)=e(k)+0.9 e(k-1)+0.385 e(k-2)
$$

in which $e(k)$ is white Gaussian noise whose variance is adjusted so that $\sigma_{z}^{2}=1$.

The averaged performances over 500 Monte Carlo runs for range, DOA, and frequency estimates of both sources are shown in Figures 2, 3, and 4, respectively, from which we can see the following facts:

(1) the proposed algorithm has a slightly worse estimation accuracy of DOA than the ESPRIT-like one at low SNR regions;

(2) although for each algorithm, the RMSE of the range estimate of source 2 (the source closer to the array) is much lower than that of source 1, our algorithm performs clearly better than the ESPRIT-like one;

(3) the proposed algorithm has satisfactory frequency estimation accuracy even at low SNR regions. (By contrast, the ESPRIT-like one assumes that the carrier frequency is known a priori.) 


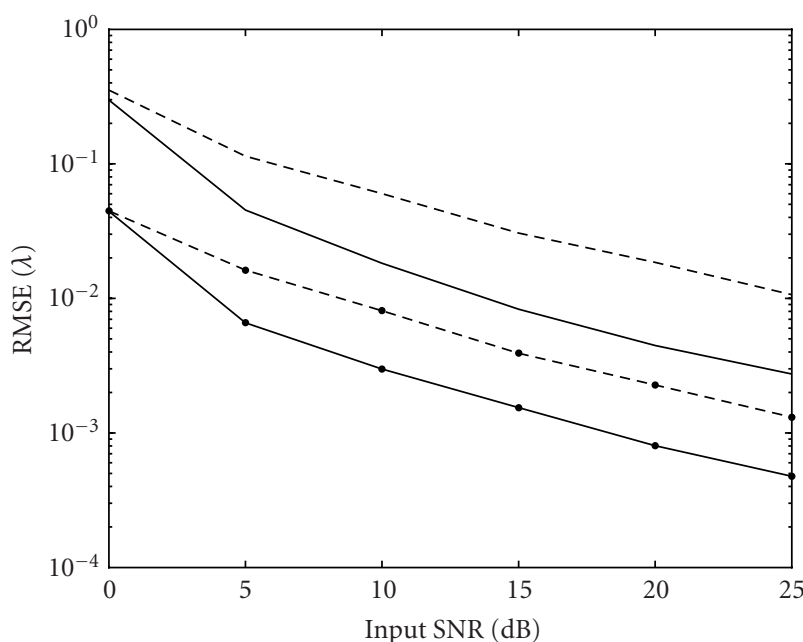

- The proposed method, source 1

$\rightarrow$ The proposed method, source 2

-... The ESPRIT-like method, source 1

-. The ESPRIT-like method, source 2

FIgURE 3: The RMSE of the estimated range over 500 Monte-Carlo runs versus the input SNR; 14 sensors and 1000 snapshots are used, and the two equi-power sources approach the array from $38^{\circ}$ and $20^{\circ}$, respectively.

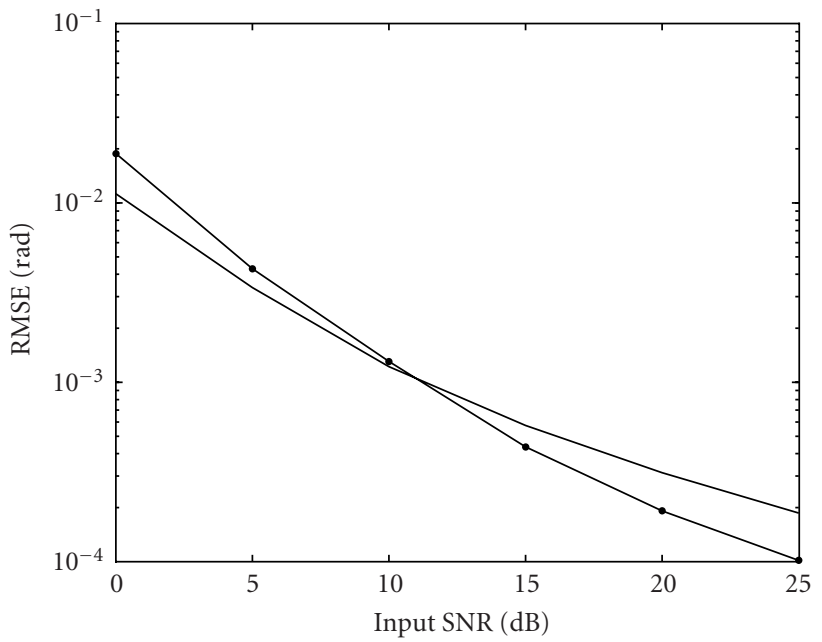

- The proposed method, source

$\rightarrow$ The proposed method, source 2

FIgure 4: The RMSE of the estimated frequency over 500 Monte Carlo runs versus the input SNR; 14 sensors and 1000 snapshots are used and the two equi-power sources approach the array from $38^{\circ}$ and $20^{\circ}$, respectively.

In the second experiment, we use the same parameters in the first experiment, except that the SNR is fixed at $15 \mathrm{~dB}$ and that the number of snapshots varies from 100 to 1900 . The

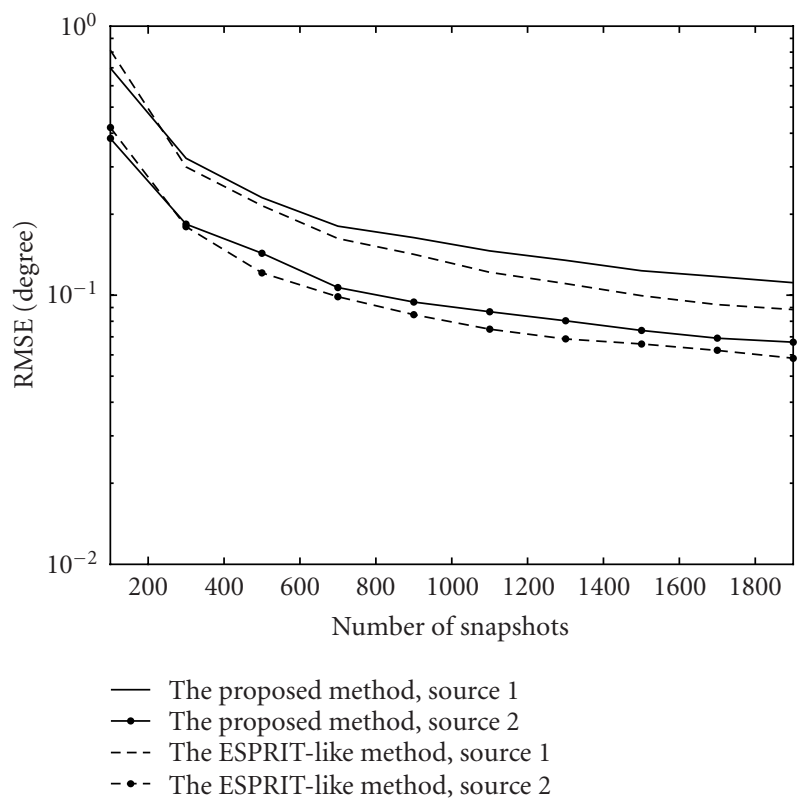

Figure 5: The RMSE of the estimated frequency over 500 Monte Carlo runs versus the number of snapshots; 14 sensors are used and the SNR is fixed at $15 \mathrm{~dB}$. Two equi-power sources approach the array from $38^{\circ}$ and $20^{\circ}$, respectively.

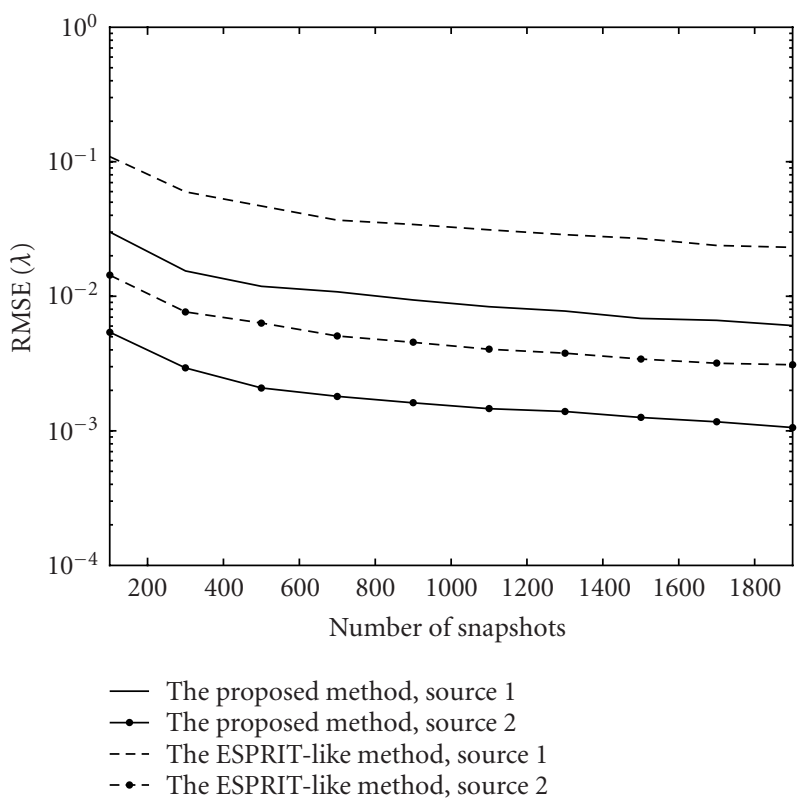

FIgURE 6: The RMSE of the estimated frequency over 500 Monte Carlo runs versus the number of snapshots; 14 sensors are used and the SNR is fixed at $15 \mathrm{~dB}$. Two equi-power sources approach the array from $38^{\circ}$ and $20^{\circ}$, respectively.

results are shown in Figures 5, 6, and 7. Obviously, similar conclusions can be made. As compared with the ESPRIT-like one [7], the proposed algorithm has greatly improved range 


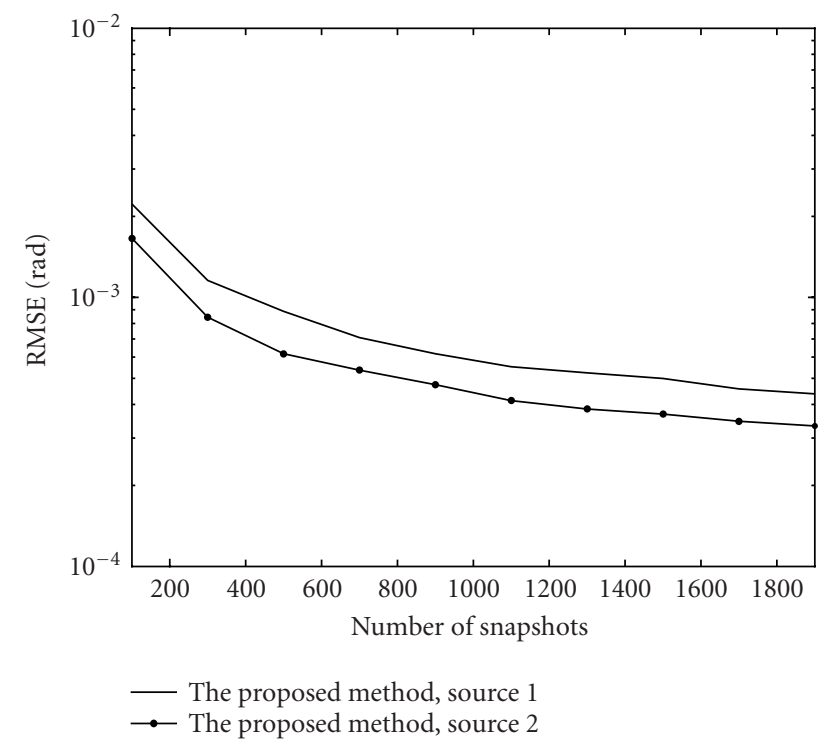

FIgURE 7: The RMSE of the estimated frequency over 500 Monte Carlo runs versus the number of snapshots; 14 sensors are used and the SNR is fixed at $15 \mathrm{~dB}$. Two equi-power sources approach the array from $38^{\circ}$ and $20^{\circ}$, respectively.

estimation accuracy since it makes full use of the information of the matrices $\mathbf{C}$ and $\overline{\mathbf{C}}$.

\section{CONCLUSION}

Based on a symmetric uniform linear array, a computationally efficient algorithm based on the fourth-order cumulants is presented in this paper for joint estimation of the range, DOA, and frequency parameters of multiple nearfield sources. The $3 \mathrm{D}$ parameters are estimated by the eigenvalues and the corresponding eigenvectors of two properly constructed matrices, and hence no additional algorithm is needed to pair among parameters. Extensive computer simulations show that the proposed algorithm performs more satisfactorily than the existing one [7].

\section{ACKNOWLEDGMENTS}

The authors would like to thank the four anonymous reviewers and the associate editor Z. Ding for their valuable comments and suggestions on the original manuscript. This work was supported by the National Natural Science Foundation of China (Grant no. 60375004).

\section{REFERENCES}

[1] H. Krim and M. Viberg, "Two decades of array signal processing research: the parametric approach,” IEEE Signal Processing Magazine, vol. 13, no. 4, pp. 67-94, 1996.

[2] A. L. Swindlehurst and T. Kailath, "Passive direction-ofarrival and range estimation for near-field sources," in 4th
Annual ASSP Workshop on Spectrum Estimation and Modeling, pp. 123-128, Minneapolis, Minn, USA, August 1988.

[3] Y.-D. Huang and M. Barkat, "Near-field multiple source localization by passive sensor array," IEEE Trans. Antennas and Propagation, vol. 39, no. 7, pp. 968-975, 1991.

[4] R. Jeffers, K. L. Bell, and H. L. Van Trees, "Broadband passive range estimation using MUSIC," in Proc. IEEE Int. Conf. Acoustics, Speech, Signal Processing, vol. 3, pp. 2921-2924, May 2002.

[5] D. Starer and A. Nehorai, "Path-following algorithm for passive localization of near-field sources," in 5th ASSP Workshop on Spectrum Estimation and Modeling, pp. 322-326, Rochester, NY, USA, October 1990.

[6] J.-H. Lee, C.-M. Lee, and K.-K. Lee, "A modified pathfollowing algorithm using a known algebraic path," IEEE Trans. Signal Processing, vol. 47, no. 5, pp. 1407-1409, 1999.

[7] R. N. Challa and S. Shamsunder, "Higher-order subspace based algorithm for passive localization of near-field sources," in Proc. 29th Asilomar Conf. Signals System Computer, pp. 777-781, Pacific Grove, Calif, USA, October 1995.

[8] N. Yuen and B. Friedlander, "Performance analysis of higherorder ESPRIT for localization of near-field sources," IEEE Trans. Signal Processing, vol. 46, no. 3, pp. 709-719, 1998.

[9] E. Grosicki and K. Abed-Meraim, "A weighted linear prediction method for near-field source localization," in Proc. IEEE Int. Conf. Acoustics, Speech, Signal Processing, vol. 3, pp. 29572960, May 2002.

[10] A. N. Lemma, A. J. van der Veen, and Ed F. Deprettere, "Analysis of ESPRIT based joint angle-frequency estimation," in Proc. IEEE Int. Conf. Acoustics, Speech, Signal Processing, vol. 5, pp. 3053-3056, Istanbul, Turkey, June 2000.

[11] G. Liao, H. C. So, and P. C. Ching, "Joint time delay and frequency estimation of multiple sinusoids," in Proc. IEEE Int. Conf. Acoustics, Speech, Signal Processing, vol. 5, pp. 31213124, Salt Lake City, Utah, USA, May 2001.

[12] T.-H. Liu and J. M. Mendel, "Azimuth and elevation direction finding using arbitrary array geometries," IEEE Trans. Signal Processing, vol. 46, no. 7, pp. 2061-2065, 1998.

Jian-Feng Chen was born in Lingbi, Anhui province, China, in 1973. He received his B.S. degree in radio electronics from the Northeast Normal University, Jilin, China, in 1996, and his M.S. degree in signal and information processing from the 54th Research Institute of China Electronics Technology Group Corporation, Shijiazhuang, China, in 1999. Currently, he is working toward his Ph.D. degree in the Key Laboratory for Radar Signal Processing at Xidian University, Xi'an, China. His research interests include array signal processing, smart antennas, and communication signal processing.

Xiao-Long Zhu received his B.S. degree in measurement and control engineering and instrument in 1998, and his Ph.D. degree in signal and information processing in 2003, respectively, both from Xidian University, Xi'an, China. Currently, he is with the Department of Automation, Tsinghua University, Beijing, China, as a Postdoctoral Fellow. His current research interests include bind signal processing, subspace tracking,

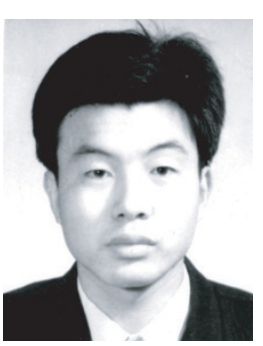
and their applications in communications.

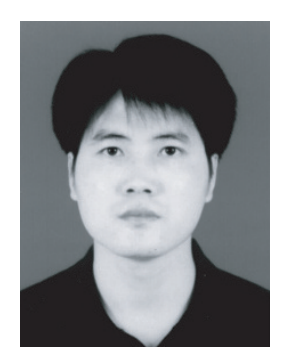


Xian-Da Zhang received his B.S. degree in radar engineering from Xidian University, Xi'an, China, in 1969, his M.S. degree in instrument engineering from Harbin Institute of Technology, Harbin, China, in 1982, and his Ph.D. degree in electrical engineering from Tohoku University, Sendai, Japan, in 1987. From August 1990 to August 1991, he was a Postdoctoral Fellow in the Department of Electrical and Computer Engineer-

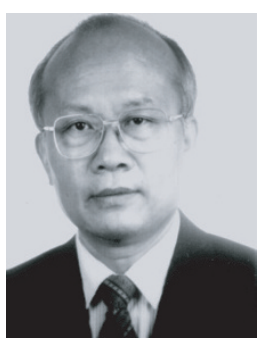
ing, University of California at San Diego. From 1992, he has been with the Department of Automation, Tsinghua University, Beijing, China, as a Professor. From April 1999 to March 2002, he was with the Key Laboratory for Radar Signal Processing, Xidian University, Xi'an, China, as a Specially Appointed Professor awarded by the Ministry of Education of China and the Cheung Kong Scholars Programme. His current research interests are signal processing with applications in radar and communications and intelligent signal processing. He has published 25 papers in several IEEE Transactions, and is the author of six books (all in Chinese). He holds four patents. Dr. Zhang is a Senior Member in IEEE and a Reviewer for several IEEE Transactions and Journals. 